\title{
Human learning in a simplified contingent discrimination situation
}

MYMON GOLDSTEIN

LONG ISLAND UNIVERSITY

Human Ss attempted to master discrimination tasks involving four displays containing nonsense forms, here schematized $a s a b, b a, c d$ and $d c$. The tasks proved difficult when position was reinforced in a way that made the distinction ab-ba vs. $c d-d c$ relevant.

Recent experiments have succeeded in establishing conditions under which human Ss fare badly when required to master discrimination tasks on the basis of stimulus position (Goldstein \& Weber, 1965; Goldstein, 1966). The evidence has been obtained with relatively uncomplicated tasks, but leaves one wondering just how simple a task design might produce similar results, i.e., methodological parsimony is at issue. The experiment reported here duplicates earlier findings with a technique of task design that has been simplified to the point of austerity.

\section{Method}

Four-display discrimination tasks were obtained by using the two conventional spatial arrangements of two pairs of stimuli. The stimuli were nonsense forms but are symbolized by letters of the alphabet in Table 1, which contains a schematic representation of the design of the experiment. Four experimental groups were employed. All groups worked with the same four displays, but the pattern of reinforcement differed from group to group as follows. In Group I-I, position was irrelevant for both pairs of stimuli, ab and cd, and stimulus identity was relevant. In Group I-L, position was irrelevant for the ab pair of stimuli which was reinforced on the basis of stimulus identity, but relevant for the cd pair of stimuli which was reinforced on the left side. In Group R-L, position was relevant for both pairs of stimuli, with reinforcement on the right side for the ab pair and on the left side for the cd pair. In Group L-L, reinforcement was on the left side for both pairs of stimuli.

The displays were prepared as 3 in. $x 5$ in. printed index cards and bound together in booklets of 384

Table 1. Schematic Representation of Experimental Design

\begin{tabular}{|c|c|c|c|c|}
\hline \multirow[b]{2}{*}{ Display } & \multicolumn{4}{|c|}{ Correct Choice } \\
\hline & Group I-I & Group I-L & Group R-L & Group L-L \\
\hline$a b$ & $a$ & a & $\begin{array}{l}\text { Right } \\
\text { (b) }\end{array}$ & $\begin{array}{l}\text { Left } \\
\text { (a) }\end{array}$ \\
\hline ba & a & $a$ & $\begin{array}{l}\text { Right } \\
\text { (a) }\end{array}$ & $\begin{array}{l}\text { Left } \\
\text { (b) }\end{array}$ \\
\hline $\begin{array}{l}\mathrm{cd} \\
\mathrm{dc}\end{array}$ & c & $\begin{array}{l}\text { Left } \\
\text { (c) } \\
\text { Left } \\
\text { (d) }\end{array}$ & $\begin{array}{l}\text { Left } \\
\text { (c) } \\
\text { Left } \\
\text { (d) }\end{array}$ & $\begin{array}{l}\text { Left } \\
\text { (c) } \\
\text { Left } \\
\text { (d) }\end{array}$ \\
\hline
\end{tabular}

Table 2. Frequencies Reaching Criterion and Error Data for Those Reaching Criterion

\begin{tabular}{lcccc} 
& \multicolumn{4}{c}{ Group } \\
\cline { 2 - 5 } & $1-1$ & I-L & R-L & L-L \\
\hline $\mathrm{N}$ & 12 & 11 & 11 & 12 \\
$\mathrm{n}$ (reached criterion) & 12 & 8 & 4 & 12 \\
$\begin{array}{l}\text { Errors to criterion } \\
\text { (n only) }\end{array}$ & & & & \\
M & 19.2 & 42.1 & 84.2 & 5.8 \\
SD & 30.7 & 41.6 & 40.8 & 3.6 \\
\hline
\end{tabular}

cards arranged in 96 successive blocks of four. Each block contained one apiece of the four displays in use. Order was random within blocks, subject to the restrictions that no display should succeed itself at a boundary between blocks, and that regular patterns of positional alternation and double alternation should not span as many as eight consecutive displays.

On each card, the correct answer was pretreated with an invisible deposit of phenolphthalein. Response was accomplished by means of a sponge-tipped moistener containing a solution of yellow vegetable dye and washing soda. As a result, incorrect choices stained yellow but correct choices stained a combination of red and yellow. The Ss, serving simultaneously in a classroom, were informed at the start that their job was to make as many correct choices as possible, i.e., they were supposed to try to maximize the number of partly red stains. In addition, they were cautioned against procedural anomalies like skipped cards and two responses per card. Despite these instructions, two of an original group of 48 Ss (12 per group) did produce such anomalies and were discarded, leaving the values of $\mathrm{N}$ reported for each group in Table 2. The Ss were male and female high school students aged 14 to 18 years and attending summer school to make up for academic deficiencies. They were distributed at random over the four experimental groups.

Results

A summary of experimental data appears in Table 2, which reports frequencies of Ss reaching a criterion of 20 consecutive correct responses and mean errors for Ss reaching criterion in each group. The frequencies and means are entirely consistent with each other and show that the most difficult treatment was encountered by Group $R-L$, which had its ab pair of displays reinforced on the right side and its cd pair of displays on the left. Next in order of difficulty was the treatment encountered by Group I-L, which had its ab pair of displays reinforced on the basis of stimulus identity 
and its cd pair of displays on the left side. The remaining two treatments caused much less difficulty, and the least difficult of all was the treatment encountered by Group L- $L$ involving reinforcement of the left side on all displays.

The intergroup differences were tested for significance by way of a median test. The resultant chisquare value was significant at well beyond the .001 level of confidence.

\section{Discussion}

It is plain that human Ss are highly capable of gross positional solutions involving a complete disregard of display appearance (i.e., Group L-L), but markedly less capable of positional solutions that are in some way dependent upon distinctions between displays. Difficulty arises when displays must be discriminated from each other on the basis of appearance (as in the present distinction of the ab pairvs. the cd pair) but then require a response governed by something other than the appearance of the chosen stimulus. In other words, much of the difficulty encountered by Groun I-L and Group R-L can be attributed to a condition of conflicting relevanceirrelevance with respect to stimulus appearance. The present results and their interpretation closely parallel the findings of Goldstein \& Weber (1965) and Goldstein (1966), obtained with somewhat different tasks.

\section{References}

Goldstein, M. Discrimination learning behavior in several situations involving intra-problem stimulus variation. Percept. mot. Skills, $1966,23,335-340$.

Goldstein, M., \& Weber, R. J. Contingent discrimination in humans Percept. mot. Skills, 1965, 21, 171-176. 